\title{
Effects of stimulus familiarity upon judged visual duration
}

\author{
LLOYD L. AVANT, PAUL J. LYMAN, and JAMES R. ANTES \\ Iowa State University, Ames, Iowa 50010
}

\begin{abstract}
Five experiments investigated the influence of the differences in stimulus familiarity among a dot-matrix letter, word, and nonword upon the relative judged duration of 30 -msec flashes. Figure-ground contrast was manipulated by varying the number of dots comprising each display letter. With 30 -msec presentations, judgments ranked subjective durations of the displays nonword $>$ word $>$ letter. Differences in judged duration among stimulus forms were greater when both forward and backward masking reduced recognition probability to chance level than when no mask was employed, and discriminations among masked presentations were easier for subjects. Figure-ground contrast influenced apparent duration judgments only as increases in figure-ground contrast contributed to clarity of familiarity differences among displays. The data were interpreted to indicate that differences in stimulus familiarity operate as early in visual processing as do differences in figure-ground contrast, with greater familiarity facilitating an automatic contact between a stimulus and its memory representation and therein reducing experienced duration.
\end{abstract}

Several writers have recently proposed cognitive theories of time perception. For example, Frankenhaeuser (1959) has suggested that subjectively experienced duration is related to the total amount of experience or "mental content" occurring within a clock-time interval. Michon (1965) emphasized the amount of information "actively coped with" in determining the experience of duration. Ornstein (1969) claimed that subjective duration is determined by the "cognitive storage size" of the processed information and "... as storage size increases, the experience of duration lengthens" (p. 41).

The studies offered by these investigators in support of their theories employed stimulus presentations of clock-time durations well above the tachistoscopic range. Ornstein, for example, suggests that the experience of duration does not accompany stimulus inputs briefer than 3 or $4 \mathrm{sec}$. However, several recent findings have indicated that, while the real-time duration of the processing of a single perceptual unit may be no briefer than $130 \mathrm{msec}$ (Efron, 1973), the apparent or subjective duration of shorter presentations is not constant. Further, the differences in apparent duration of tachistoscopic presentations may index the operations of perceptual or preperceptual processing. When Liss (1968) presented $1-, 2-$, or 4-letter arrays for real-time durations ranging from 7 to $100 \mathrm{msec}$, his subjects volunteered

This research was supported in part by Grant $\mathbf{M H} 24964$ from the National Institute of Mental Health to the first author and in part by NSF Grant GU 3373 to Iowa State University. We wish to thank Marilyn Eshelman and Tim McGaughey for assistance in collecting the data of Experiments I and III. Requests for reprints should be sent to Lloyd L. Avant. Department of Psychology, Iowa State University, Ames, Iowa 50010 . J. R. Antes is now at the University of North Dakota, Grand Forks, North Dakota 58201. reports that arrays presented for $70 \mathrm{msec}$ and followed by a noise mask appeared to be of shorter duration than equivalent arrays presented for $7 \mathrm{msec}$ without the mask. Similarly, Sperling (1967) found that, when real-time duration of stimulus arrays was near threshold for letter recognition, a $10-\mathrm{msec}$ increase in exposure duration produced an increase in apparent duration of considerably more than $10 \mathrm{msec}$.

While the reports of Liss's (1968) and Sperling's (1967) subjects may reveal the influence of such variables as apparent brightness (though Liss's subjects found brightness judgments extremely difficult to make under his conditions) or apparent figure-ground contrast, Buffardi (1971) has shown that such differences in apparent duration occur in the auditory and tactual modalities as well as in vision. For all three modalities, Buffardi presented 96-msec stimulus inputs, and the number of inputs varied from 0 to 5 over a total observation interval of $1,056 \mathrm{msec}$. Results were similar in all modalities; apparent duration of the total observation interval increased with number of stimulus inputs, with the greater influence coming from stimuli occurring early in the observation interval. Buffardi interpreted his results as support for Ornstein's "cognitive storage size" theory.

Observations volunteered by subjects in a study of letter detection in the writers' laboratory suggest that differences in the apparent durations of tachistoscopic presentations may index the influence of such cognitive variables as stimulus familiarity, as well as the influence of apparent brightness or contrast, upon early visual processing. In that study (Avant. Note 1), stimulus materials were single vowels and CVCs, each presented for $30 \mathrm{msec}$. The subjects reported that the apparent durations of the $30-\mathrm{msec}$ flashes varied, 
depending on how degraded the stimulus forms were and on whether single vowels or CVCs were presented. Since, for many of the stimulus forms. letter detection was no better than chance level. the differences in apparent duration appeared to be related to stimulus parameters which exert their influence on processing before the subject can identify the stimulus. The experiments to be reported here were designed to determine whether differences in the apparent durations of tachistoscopic presentations were systematically related to such cognitive variables as stimulus familiarity as well as. or instead of, such perceptual variables as apparent brightness or apparent contrast.

\section{EXPERIMENT I}

\section{Method}

Stimulus materiak. Experiment I was exploratory in nature and uas designed to determine whether apparent duration judgments were systematically related to stimulus forms like those employed in the study of letter detection (Avant, Note 1). Stimuli for that study consisted of single vowels and CVCs, formed by the cells of $21 \times 34$ cell computer printout matrices. The program that generates the stinuli identities, within each matrix, the cells which. when filled, form sartous letters of the alphabet. On successive runs through the program. an asterisk is printed in a randomly selected $1 \%$ of the cells forming each letter. These mutually exclusive $1 \%$ portions of the possible cells in each letter are accumulated across runs of the program, permitting variation in the information specifying each ietter from 1 to $100 \%$ filled cells. These computer printouts were converted to $2 \times 2$ in. positive transparencies for presentation in a Scientilic Prototype Model GB tachistoscope. When vieued in the tachistoscope. the slides appeared as letters comprised of dots.

In the study of letter detection, stimuli were single rowels and $100 \%$ association value CVCs (three-letter words), each printed and photographed with $15 \%, 30 \%$, and $45 \%$ of the cells in each letter filled. Since subjects in the detection study reported that $30-\mathrm{msec}$ presentations of those stimuli varied in apparent duration, the stimulus forms selected for Experiment I were the vowel I and the word HIT, each printed and photographed with $15 \%, 30 \%$, and $45 \%$ of the cells in each letter matrix filled. Since responses to such stimuli might not be representative of responses subjects would make to fully formed letters and words, slides were also made of I and HIT cut from black construction paper to match computer printout size. Viewed monocularly in the tachistoscope, the vertical and horizontal visual angles subtended by the $I$ were $1^{\circ} 13^{\prime}$ and $27^{\prime}$. respectively; for HIT, these visual angles were, respectively, $1^{\circ} 13^{\prime}$ and $1^{\circ} 37^{\prime}$. All stimuli were presented with luminance levels of the tachistoscope fields adjusted to project, with no stimuli in the fields, $6.3 \mathrm{fL}$. as measured by a Spectra Model UB $1 \frac{1 / 2}{2}$ brightness spot meter.

Design and Procedure. The design and procedure were suggested by Ornstein's (1969) contention that, to investigate the experience of duration. the appropriate measure should index the relative experienced duration of two intervals. Thus, "duration comparisons [should be] made directly between the relative experiences of two intervals" (Ornstein, 1969, p. 51), not between the experienced duration of an interval and a temporal unit defined by the clock.

In accord with Ornstein's argument. the two stimulus forms (I and HIT) and four information levels $(15 \%, 30 \%$, and $45 \%$ filled cells plus solid black letters) were combined factorially to generate an eight-member stimulus set, and the eight stimuli of the set were presented for judgment by the method of pair comparisons. Each combination of stimulus form and information level was paired with. and judged against. every other combination of these two variables. resulting in $\mathrm{N}(\mathrm{N}-1), 2=28$ judgments of stimulus pairs. On each trial of the experiment, the two stimuli of that pair were presented in Fields 1 and 3 of the tachistoscope while the blank field was viewed during the 1 sec separating members of the pair. The blink field was also viewed during the interval between trials of the experiment (approximately $10 \mathrm{sec}$. A ready signal cued the subject to attend to the center of the blank $3.5^{\circ}$ vertical $\times 6.5^{\circ}$ horizontal field immediately before the experimenter initiated each pair of stimulus tlashes. Presentation of each stimulus as the first or second member of each pair was equalized across the experiment. and the orders for judging the 28 stimulus pairs were randomly arranged and varied across subjects.

To begin the experiment. each subject was informed that he would be seeing two brief stimulus flashes. separated by a somewhat longer blank interval. and that he was to report. on each trial. which of the two stimulus tlashes appeared to be of longer duration. He was shown the $45 \sigma_{0}$ level slide of 1 and HIT in one field of the tachistoscope for 2 or $3 \mathrm{sec}$. and he was presented 5 randomly selected pairs as practice trials. Subjects were not told that information level would vary. Both stimuli of each pair were presented for $30 \mathrm{msec}$, and the members of each pair were separated by a $1 . \mathrm{sec}$ presentation of the blank field. The 28 stimulus pairs were presented once to each subject. and the measure reflecting relative judged duration for each of the eight stimuli was the $z$ ' score corresponding to the proportion of "longer" responses given each stimulus by each subject (see Woodworth \& Schlosberg. 1954. pp. 252-254. for derivation of $z^{\prime}$ scores). These $z^{\prime}$ scores were evaluated in a balanced within-subjects analysis of variance. with stimulus form and information level considered fixed factors while subjects were random.

Subjects. Twenty-five students of introductory psychology served as subjects and received course cred it for research participation. All subjects reported normal or corrected-to-normal vision.

\section{Results and Discussion}

As is evident in the plot of average $z^{\prime}$ score for each stimulus in Figure 1, the judged duration of the $30-\mathrm{msec}$ stimulus flash increased reliably with increases in percent filled cells (PCF) identifying each stimulus form $[F(3.72)=84.89, \mathrm{p}<.001]$, and presentations of HIT were consistently judged to be of

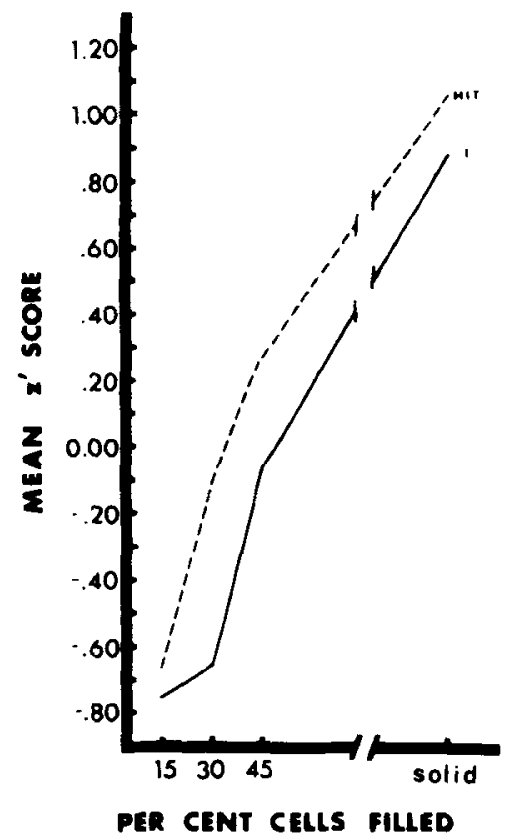

Figure 1. Mean $z$ scores retlecting relative judged daration of 30-msec flashes of I and BIT with stimulas forms specified by 15-45 PCF or by solid black letters (Experiment $D$ ). 
longer duration than equal presentations of $I[F(1,24)$ $=14.26, \mathrm{p}<.001]$. The interaction between stimulus form and PCF was not significant; the lack of an interaction suggests that responses to dot matrix stimuli and to solid black fully formed stimuli were not generated by grossly different processes.

In demonstrating that the judged duration of $30-\mathrm{msec}$ flashes was systematically related to both independent variables, Experiment $I$ achieved its major purpose. However, in terms of identifying the processes responsible for the differences in judged duration, the data do little more than suggest testable hypotheses. At least the following hypotheses, some of which are not independent of each other, might be considered in addressing the data.

(1) Since increases in either PCF or number of letters would augment figure-ground contrast, the influence of both independent variables might be attributable to apparent contrast.

(2) Since the retinal area stimulated by HIT was larger than that for I, the stimulus form effect may simply reflect the subjects' detection of a difference in stimulus area.

(3) Assuming that subjects extracted more information than a simple discrimination of either apparent contrast or stimulus area, the difference between I and HIT may reflect partial extraction of one vs. three letters.

(4) Assuming that no differences in subjective duration could occur when all stimulus presentations were for $30 \mathrm{msec}$, when the subjects were forced to decide between two flashes on each trial, the basis for the subjects' decision between stimulus forms may have been a differential ease with which stimulus forms could be recognized. Several studies of the word-letter phenomenon (e.g.. Reicher, 1969; Wheeler, 1970) have shown the letters of words to be more readily recognized than the same letters presented alone. Thus, the data of this experiment may indicate that, when subjects were forced to discriminate between a pair of flashes when no subjective differences could occur, they concluded that the stimulus form which was more easily recognized must have been presented for a longer time.

(5) A fifth possible account of the results of this experiment might suggest that a difference in the familiarity of the two stimulus forms operated at this early stage of processing to generate differences in the subjective durations of the two stimulus forms. Such an account would hold that the difference in apparent durations for the two stimulus forms resulted becaluse the greater familiarity of I facilitated the initial extraction of information from that display relative to that for the HIT display and resulted in a shorter subjective duration. Support for this hypothesis would indicate that differences in stimulus familiarity operate as early in visual processing as do differences in apparent contrast.
The succeeding experiments of this report were designed to more clearly differentiate among these possible accounts of the differences in apparent duration that accompany tachistoscopic presentations.

\section{EXPERIMENT II}

Experiment II was designed to more clearly index the intluence of stimulus familiarity upon judged duration when stimulus area was controlled and when ease of stimulus recognition was manipulated in a noise mask vs. no-mask comparison. The following rationale dictated the operations of Experiment II.

If the major determinant of apparent duration of tachistoscopic flashes is apparent contrast, stimulus area, or number of stimulus letters, then, in Experiment II, if stimulus forms are I, DIG, and EIO, judged durations for DIG and EIO should be the same and these should differ from the judged duration of $I$. However, if subjects judge the flash presenting the more easily recognized display to be longer, Reicher's (1969) and Wheeler's (1970) results suggest that presentations of DIG should be judged to be longer than presentations of $I$ and EIO. Further, if $30-\mathrm{msec}$ presentations of these forms are judged against each other when a noise mask both precedes and immediately follows each stimulus presentation as well as when no mask is presented, the ordering of judged durations for the three stimulus forms should be the same under both mask conditions unless the mask differentially influences the recognizability of the three forms.

To the extent that subjects judge the more easily recognized stimulus to be of longer duration, additional expectations of the influence of a noise mask may be specified. Since, with a noise mask both preceding and immediately following each stimulus flash, both forward and backward masking should reduce recognizability of stimulus forms (see Kahneman, 1968; Lefton, 1973; Raab, 1963; Turvey, 1973), then any differences in judged durations for the three forms should be less clearly drawn under the masked condition than under the no-mask condition. Further, when subjects must make a forced choice between flashes on each trial, recognizability should be reduced by the mask, and the difficulty of making the decision should be greater for the masked than for the no-mask condition (irrespective of the stage of processing at which the mask has its interfering effect). The greater decision difficulty for the masked condition should reflect itself in longer response latencies for the masked condition.

Different expectations may be derived from the hypothesis that differences in judged duration are generated by very early stages of visual processing with the influence of stimulus familiarity operating at these early stages to make the less familiar stimulus appear to be of longer duration. If stimulus familiarity operates at the initial stages of visual 
processing so as to facilitate the processing of familiar stimuli relative to that for unfamiliar stimuli and result in shorter apparent durations for the more familiar stimuli, then the expected ordering of judged durations would be EIO greater than DIG greater than 1 . Further, if differences in apparent duration generated by the influence of familiarity at initial stages are passed or washed out by subsequent processes as full blown recognitions occur, then one might expect the masked condition to more precisely limit processing to initial stages and permit clearer separations among stimulus forms for the masked than for the no-mask condition.

\section{Method}

Stimulus materials. As in Experiment I. stimulus materials were formed by the cells of $21 \times 34$ cell computer printout matrices. 1 , DIG. and EIO were the forms, each printed and photographed with $15 \%, 30 \%$. and $45 \%$ of the cells filled. For the masked condition. these combinations of stimulus form and PCF were presented in Fields 1 and 3 of the tachistoscope. During the 1-sec separation of the members of each stimulus pair, a noise mask containing asterisks in all cells of the rectangular area defined by the three-letter matrices was presented in the other field of the tachistoscope. This field was viewed during the interstimulus interval as well as during the interval separating trials of the experiment. For the no-mask condition, the blank field of the tachistoscope was empty except for four small right angles marking the corners of the rectangular stimulus area. Visual angles of stimulus forms and light levels of the tachistoscope were as described for Experiment 1 .

Design and Procedure. The three stimulus forms (I, DIG, and EIO) were combined factorially with three PCF levels $(15 \%, 30 \%$, $45 \%$ cells filled) to form a nine-member stimulus set. As in Experiment I, the method for judgment was pair comparisons, with the $(9 \times 8) / 2=36$ comparisons of each combination of stimulus form and PCF against every other combination being judged by each subject once under the masked condition and once under the no-mask condition. For half the subjects, the stimulus set was judged first under the masked condition and then under the no-mask condition; for the remaining subjects, the order of the two mask treatments was reversed. With the exception that the subjects were informed that they would be judging under both mask treatments. instructions and practice trials were as described for Experiment $I$. To assure a subject's maximum concentration on the task. the procedure of Experiment I was modified to permit the subject to initiate presentation of each stimulus pair. On each trial, a 1 -sec delay intervened between the subject's switch closure to initiate the trial and the appearance of the first stimulus flash. The $z$ scores reflecting relative judged duration for each stimulus by each subject were derived as described for Experiment $I ; z^{\prime}$ scores were separately calculated for the mask and the no-mask treatment. A single-pole double-throw switch was mounted directly below the tachistoscope eyepiece, and the subjects indicated that the first or second flash of each pair was longer by a right or left throw of that switch; response latencies were recorded, to the nearest millisecond, by a Hunter Model 120A Klockounter. The $z$ ' scores were evaluated in a balanced analysis of variance, with order of judging under the mask and no-mask conditions the between-subjects variable and mask treatment, stimulus form, and PCF the within-subjects variables. Since no stimulus presented under the masked condition was judged against any stimulus presented under the no-mask condition. derivation of $z^{\prime}$ scores for each mask treatment was constrained to the separate applications of the pair comparisons procedure. To ascertain the effect of stimulus form and PCF for each mask treatment, unconstrained by the effects of these two variables under the other mask treatment, two additional analyses. were performed. These analyses evaluated $z^{\prime}$ scores for each mask treatment independently; mean separation tests reported below were performed using error terms (confined to the separate determinations of $z^{\prime}$ scores) from these separate analyses.

\section{Results and Discussion}

Results of these analyses confirmed and extended the results of Experiment $l$. In the overall analysis, neither the main effect of the two orders for judging under the two mask treatments nor any interaction involving order was significant. The main effect of mask treatment was not significant, while the main effects of stmulus form $[\mathrm{F}(2,44)=22.09, \mathrm{p}<.01]$ and of PCF $[F(2,44)=433.09, \mathrm{p}<.001]$ were significant. The most informative finding with $z^{\prime}$ scores was the significant three-way interaction among mask treatment, stimulus form, and PCF $[\mathrm{F}(4,88)=3.49, \mathrm{p}<.05]$, which is plotted in Figure 2.

Two findings are of particular importance in viewing the data presented in Figure 2. First, under both mask conditions, judged duration for the three stimulus forms ranked them, from longest to shortest, EIO, DIG, and I. Applied to the main effect of stimulus form under the masked condition, Scheffé's test $(a=.05)$ showed that, on the average, presentations of EIO were judged to be longer than equal presentations of DIG, which were judged to be longer than equal flashes of $I$. Under the no-mask condition, the reliable differences in judged durations of stimulus forms were between EIO and DIG and between EIO and I; the difference between DIG and I was not significant. For both mask treatments, differences among the three PCF levels were all significant.

Second, the differences among judged durations of the three stimulus forms were more clearly drawn

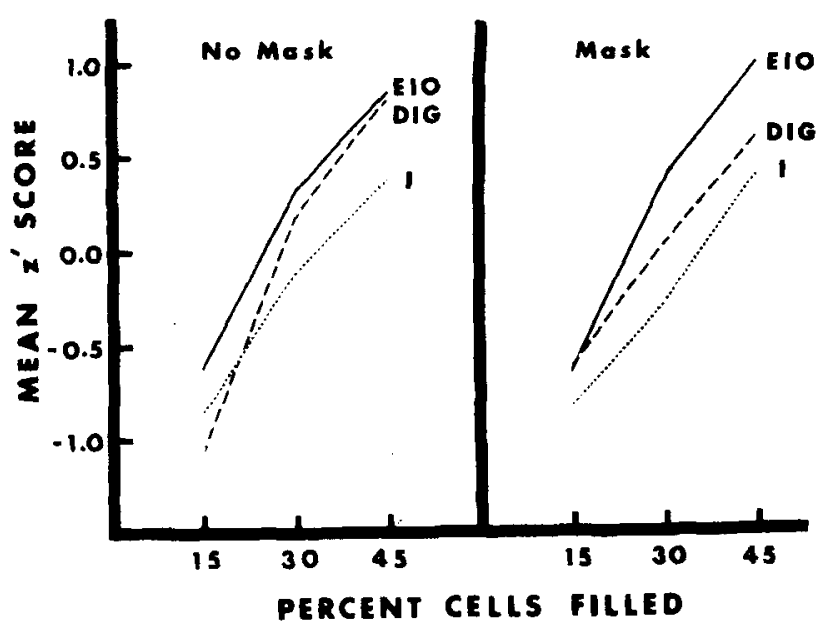

Figure 2. Mean $z^{\prime}$ scores reflecting relative judged duration of 30-msec flashes of I, DIG, and EIO when each stimulus form was specified by 15,30 , or 45 PCF. The right panel reflects judgments when each stimulus was preceded and followed immediately by a dot noise mask; the left panel presents judgments of unmasked flashes (Experiment II). 
under the masked condition than under the no-mask treatment. The clearer separation for the masked condition was evident in the main effect of stimulus form and the interaction between stimulus form and PCF. Under the masked condition. Scheffe's test revealed that no differences among stimulus forms occurred when onls $15 \%$ of the cells in each letter were filled. At both the $30 \%$ and the $45 \%$ levels of PCF. the reliable differences were between EIO and I while DIG differed from neither EIO nor I. A comparable test of the differences between stimulus forms at each PCF level for the no-mask condition revealed no significant differences.

The $z^{\prime}$ scores of Experiment II convincingly demonstrate that the judged durations of 30 -msec presentations of these stimulus forms reflects the processing of more than just apparent contrast, retinal area of stimulation, or number of letters. The longest-to-shortest ranking of judged durations of the three forms. from the unfamiliar letter string (EIO) to the more familiar word (DIG) to the even more familiar letter (I), and the fact that differences among judged durations of the three forms were clearer for the mask than for the no-mask condition suggest that the differences in judged duration reflect the influence of differential stimulus familiarity at very early stages of visual processing. According to Sternberg's (1969) interpretation of interactions between independent variables. the three-way interaction among stimulus form. PCF. and mask treatment indicates that stimulus familiarity and PCF are operating at the same level of processing and that the effects of their operation are clearer to the subject when presentations are masked.

Response latencies. Reciprocals of the latencies of subjects' responses to respective pairs of stimulus configurations were subjected to four separate analyses. Since each response was to a pair of different stimuli, these analyses could not be totally independent of each other. In these analyses. the measure was collapsed across judgments of either stimulus form or PCF. In the first analysis, the measure was collapsed across levels of PCF, and the influence of judging I vs. DIG, I vs. EIO. and DIG vs. EIO was evaluated under both mask treatments. The second analysis was like the first except that the measure was collapsed across stimulus forms and it evaluated the influence of judging $15 \%$ vs. $30 \%, 15 \%$ vs. $45 \%$, and $30 \%$ vs. $45 \%$ PCF levels under the two mask treatments. These two analyses evaluated the intluence of the two independent variables when the members of the judged pair were different. either in stimulus form or in PCF. Two comparable analyses were performed to evaluate the influences of these variables when the members of the judged pair were the same, either in stimulus form or PCF (i.e.. I vs. I. DIG vs. DIG. EIO vs. EIO or $15 \%$ vs. $15 \%, 30 \%$ vs. $30 \% .45 \%$ vs. $45 \%$ ). In each analysis, the measure consisted of the mean of the reciprocals of each subject's response latencies for judging each of the
Table 1

Mean Response Latencies (Milliseconds) for Judgments of Each Stimulus Form and PCF When Each Level of These Two Variables Was Judged Against the Same Level and Agrinst a Different Level of the Same Variable

\begin{tabular}{|c|c|c|c|c|c|}
\hline & Mask & $\begin{array}{c}\text { No } \\
\text { Mask }\end{array}$ & & Mask & $\begin{array}{c}\text { No } \\
\text { Mask }\end{array}$ \\
\hline \multicolumn{3}{|c|}{ Stimulus Forms: Different* } & \multicolumn{3}{|c|}{ PCF: Different* } \\
\hline $\begin{array}{l}\text { I vs. DIG } \\
\text { I vs. EIO } \\
\text { DIG vs. EIO }\end{array}$ & $\begin{array}{l}929 \\
872 \\
851\end{array}$ & $\begin{array}{l}955 \\
988 \\
910\end{array}$ & $\begin{array}{l}15 \text { vs. } 30 \\
15 \text { vs. } 45 \\
30 \text { vs. } 45\end{array}$ & $\begin{array}{l}858 \\
796 \\
911\end{array}$ & $\begin{array}{l}924 \\
855 \\
961\end{array}$ \\
\hline \multicolumn{3}{|c|}{ Stimulus Forms: Same† } & \multicolumn{3}{|l|}{ PCF: Same $\dagger$} \\
\hline I vs. I & 914 & 940 & .15 vs. 15 & 941 & 1032 \\
\hline DIG vs. DIG & 843 & 902 & 30 vs. 30 & 919 & 1010 \\
\hline EIO vs. EIO & 820 & 912 & 45 vs. 45 & 978 & 1054 \\
\hline
\end{tabular}

*Each mean based on nine responses per subject, 24 subjects tEach mean based on three responses per subject, 24 subjects.

stimulus comparisons being evaluated. All four analyses were balanced analyses, with mask vs. no mask the between-subjects variable and stimulus form or PCF the within-subjects variable. Except for subjects. all factors were considered fixed.

These analyses showed that. when the two flashes of a trial presented configurations which were the same in either stimulus form or PCF, neither of these two variables differentially influenced response latency. However, latencies of judging comparisons involving different stimulus forms differed significantly $[F(2,46)$ $=3.74 . \mathrm{p}<.05]$, as did latencies for judging comparisons involving different PCF levels $[F(2,46)=$ 10.25. $p<.001]$. The most informative results of these analyses appeared in the mask vs. no-mask comparisons. Since the masked condition should deter stimulus recognition, the hypothesis that longer apparent duration accompanies the more easily recognized stimulus also predicted longer response latency for the masked condition. The hypothesis that duration judgments reflect prerecognition influences of stimulus familiarity predicted the opposite result: shorter response latency for the condition under which processing was confined to early stages by the mask. While the mask vs. no-mask differences in response latency were not large enough to reach an acceptable level of statistical significance in the analyses, the direction of the differences clearly favored the latter hypothesis. As Table 1 show's, mean latencies for all comparisons were numerically smaller for the masked condition. As was noted above. the four analyses of the latency measure could not be totally independent of each other. However, the analyses were not totally dependent either and it should be noted that. were the measures totally independent. the probability of obtaining the 12 mask vs. no-mask differences of Table 1 all in the same direction would approximate .0005 .

The response latency results were, then, entirely compatible with the judged duration measure. The apparent duration judgments showed clearer differentiations among stimulus forms under the 
masked condition than the no-mask condition. The lower response latencies for the masked condition also indicates that the differences in apparent duration were clearer for the subjects under the masked condition: apparently, the subjects found it less difficult to discriminate between subjective durations when processing of the stimulus flashes was limited to early stages by the mask. This eridence. combined with the EIO $>$ DIG $>$ I ordering of judged durations. suggests strongly that the differences in judged duration are indexing the influence of stimulus familiarity upon rery early visual processing.

\section{EXPERMENT III}

Considered collectively. the results of the first two experiments suggest that stimulus familiarity intluences the very earliest stage of risual processing. However. this conclusion is based on indirect tests of the recognizability of these stimulus forms-i.e.. the mask is. no-mask comparison of judged duration and the mask is. no-mask comparison of response latencies. Experiment Ill directly tested the recognizability of the stimulus forms employed in Experiment II under the conditions of that experiment.

\section{Method}

Stimulus materials. In order to present a two-alternative furced-choice test of the recognizability of the Experiment II stimulus forms, stimulus materials for Experiment III included I. DIG. and EIO from Experiment II and added O, DOG, and $\triangle O \Delta$, each printed and phototgraphed with $30 \%$ and $45 \%$ cells filled. All stimuli were generated by the computer program described in Experiment I.

Design and Procedure. As in the first two experiments, the six stimulus forms (1. DIG, EIO, O, DOG, $\triangle O \Delta$ ) and two PCF levels $(30 \%$ and $45 \%$ cells filled) were combined to generate a set of 12 stimuli which was presented in two separate series of $(12 \times 11): 2=$ bo paired presentations of each stimulus once with every other stimulus. For each subject, the order of the 66 pairs in each presentation of the set was independently randomized. For the first presentition of the stimulus set. the appearance of each stimulus as the first or second member of the pair was determined randomly; in the second presentation of the set, each stimulus $w$ as presented as the opposite member of the pair. For one group of subjects, these tho presentations of the stimulus set were under the no-mask condition of Experiment 1I; for the other group, presentations were under the masked condition of Experiment II. Exposure duration and intersignal interval. luminance levels of the tachistoscope, and visual angles subtended by the stimuli were as described for Experiments I and II. Subjects were not told what the stimulus forms would be but were given five randomly selected pairs of stimuli is practice trials.

Instid of reporting which one of the paired 30 -msec flashes was apparently longer. the subjects were asked to report, after each pair of flashes. whether an I or an $\mathrm{O}$ had been presented in each flash of the pair. Thus, the performance measure indexed the accuracy with which individual letters of the stimulus arrays could be verbally identitied under the conditions $x$ hich prompted the differences in judged duration in Experiment II. For each subject, the probability of correct report of $I$ and $O$ in each stimulus array at each PCF level when each stimulus was presented as the first and as the second member of each pair was transformed to 2 arcsin $\sqrt{X}$ (Winer, 1971. pp. 399.400). These transformed scores were evaluated in a balanced analysis of variance: mask vs. no mask was the between-subjects variable. while stimulus form. PCF, vowel, and first or second flash were within-subjects variables. All factors. except subjects. Were considered fixed.

Subjects. Six volunteers from introductory psychology courses were assigned randomly to two groups of three subjects and were given course credit for research participation. All subjects had normal or corrected-to-normal vision.

\section{Results and Discussion}

The results of the recognition measure of Experiment III complemented the judged duration measure of the first two experiments in indicating that stimulus familiarity influenced apparent duration well before stimulus forms could be recognized. In Experiment II. differences in apparent durations of I. DIG. and EIO were more clearly drawn under the masked than under the no-mask condition; in Experiment III. average probability of correct letter recognition was .81 for the no-mask condition and .52 for the masked condition $[F(1.4)=11.74 . p<.05]$. While the longest-to-shortest ranking of apparent durations for stimulus forms in Experiment II was EIO. DIG. and 1 . recognition probabilities in Experiment III ranked them in the opposite order: .80 for single letters (I. O). .65 for words (DIG, DOG), and .57 for nonwords (EIO. $\Delta \mathrm{O} \Delta)[F(2,8)=$ 9.97. $\mathrm{p}<.01]$. For the no-mask condition, probability of accurate report was .76 for the $30 \%$ PCF level and .87 for the $45 \%$ level; probability of correct identification was .52 for both PCF levels under the masked condition $[F(1.4)=26.08$. $\mathrm{p}<.01]$. Thus, the masked condition produced clearest separations in judged durations of stimulus forms in Experiment II and chance level recognition in Experiment III. Finally, while subjects differed in orerall lerels of report accuracy. the effects of mask conditions nere consistent across subjects: for the three subjects of the no-mask treatment, probabilities of accurate report were $.66, .88$, and .88 ; these probabilities for the subjects of the masked condition were $.50, .50$, and .56 .

Collectively, the results of the first three experiments argue that judgments of the apparent duration of tachistoscopic presentations index the influences of figure-ground contrast and stimulus familiarity upon very early visual processing. These data suggest that. to initiate processing of stimulus forms. some minimum level of information must specify the form to be processed; no differences in apparent duration occurred when only $15 \%$ of the cells in stimulus forms were filled, and as PCF increased to $45 \%$, judged durations of stimulus forms progressively diverged.

\section{EXPERIMENT IV}

In the first three experiments. figure-ground contlast was determined by PCF, and in these experiments. this variable had a very strong effect. Howerer, the duration judgment tasks of Experiments 
1 and II did not permit determination of whether judgments of apparent duration differentially index the influence of contrast independently of any effect of stimulus familiarity. It is also to be noted that these experiments employed naive subjects who were presented relatively few trials. And. while the systematic functions for stimulus form and PCF do not recommend it, the possibility remains that the duration judgments in these experiments were an artifact-some peculiarity of early experience in making tachistoscopic judgments with the pair comparisons procedure. Experiment IV was designed to determine the influence of simple contrast on duration judgments and the effects of practice with tachistoscopic judgments.

\section{Method}

Stimulus materials. Subjects in this experiment performed two time judgment tasks. Stimulus materials for the first task eliminated any influence of stimulus familiarity upon duration judgments by presenting only randomly dispersed dots. To generate stimuli for this task, the number of dots comprising I, DIG, and EIO at each PCF level was counted. These counts showed $I-15 \%=$ 63. $1-30 \%=126.1-45 \%=189$. DIG $-15 \%=232$. DIG $-30 \%$ $=464 . \mathrm{DIG}-45 \%=696$. EIO $-15 \%=224$, EIO-30\% $=448$, and EIO- $-45 \%=672$ dots. For the first task. nine slides were prepared containing the above numbers of dots randomly dispersed over the rectangular areas corresponding to a single letter or a three-letter array. These slides closely approximated the apparent contrast generated by the stimulus forms of Experiment II but removed any familiarity effect of meaningful verbal arrays. For the second task, stimulus materials were the $15 \%, 30 \%$, and $45 \%$ PCF level slides of I. DIG, and EIO from Experiment II. All stimuli were generated by the computer program described in Experiment $I$, and visual angles of stimulus forms, light levels of the tachistoscope, and exposure durations matched those of the earlier experiments.

Design and Procedure. With the exception that subjects were told nothing about stimulus materials. the design and procedure duplicated those of the first experiment. The 36 paired comparisons of each task paired each stimulus against every other stimulus, and subjects judged which of the paired flashes was of longer apparent duration. $z$ scores for each stimulus for each subject were derived by the same procedure. and they were evaluated by analysis of variance. For the first task. stimuli were treated as three levels of PCF and three stimulus forms; the first form was considered to be the three slides presenting the three PCF levels of I, the second the three slides presenting the PCF levels of DIG, and the third the three slides presenting the PCF levels of EIO. All stimuli were judged under the masked condition of Experiment II, and z' scores were evaluated in a balanced within-subjects fixed effects analysis of variance. Except that subjects were not told what the stimulus forms would be, the second task exactly replicated the masked treatment of Experiment 1I.

Sabjects. Twenty volunteers from summer session psychology courses served as subjects and were given course credit for participation. All subjects had normal or corrected-to-normal vision.

\section{Results and Discussion}

Analysis of $z^{\prime}$ scores from the first task showed that apparent contrast did not influence duration judgments independently of form differences. The only statistically significant effect was due to stimulus forms $[F(2.38)=5.23, p<.05]$, which, in this experiment. were simply differences in the area covered by the rectangular random dispersion of dots. The $F$ value for PCF was less than 1.00 . and there was

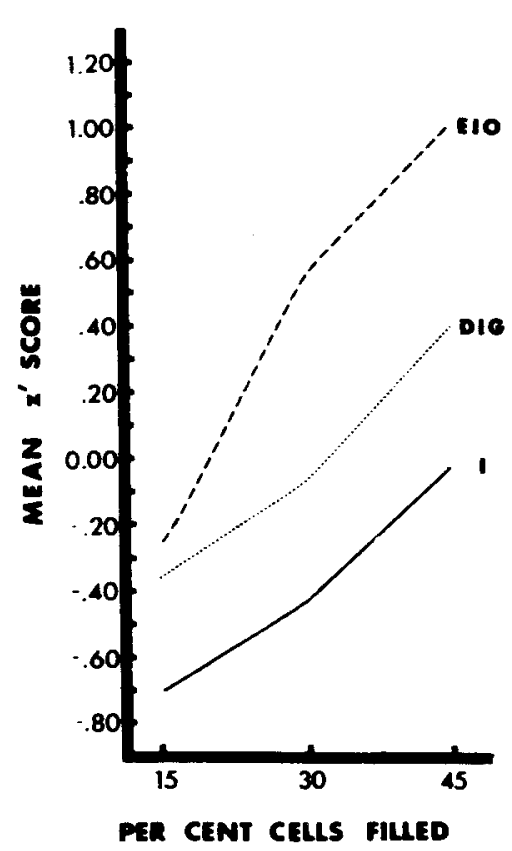

Flgure 3. Mean $z$ score for each atimulus form at each PCF level in the recond task of Experiment IV.

no interaction between stimulus form and PCF. Scheffé's test $(a=.05)$ showed the three-letter areas to be judged to be of longer duration than the one-letter areas, but duration judgments for the three-letter areas did not differ. Compared to the first two experiments, in which contrast was determined by the number of dots comprising each stimulus fornil. the contribution of PCF to apparent contrast had no influence when contrast was devoid of any meaningful form other than size of the stimulus area. These results indicate that, while differences in subjective duration are not sensitive to differences in simple contrast, they are sensitive to the initial establishment of differences in size of the presented stimulus.

The data from the second task of this experiment clearly demonstrate that differences in apparent duration for stimuli of varying familiarity are not artifacts of initial experience in judging tachistoscopic flashes. When, following the time judgments of the tirst task. subjects were asked to judge the apparent durations of I. DIG, and EIO under the masked condition of Experiment II. the results showed a clear replication of the earlier data. Mẹan relative judged durations for each stimulus form at each PCF level are presented in Figure 3. Experience with judging tachistoscopic flashes gained in the first task appeared to make the influence of both variables, as well as their interaction, slightly stronger. The main effect of stimulus form was significant $[F(2,38)=$ 43.5. $p<.001]$, as was the main effect of PCF $[F(2,38)=93.7, p<.001]$. Applied to the significant interaction between these variables $[F(4,76)=3.80$. $p<.01]$. Scheffés test showed no reliable differences in judged duration among stimulus forms when only 
$15 \sigma_{0}$ of the cells were tilled; at $30 \%$ filled cells. ElO was judged reliably longer than I, while DIG appeared to be of an intermediate duration that differed from neither EIO nor $\mathrm{I}$. and at $45 \%$ filled cells. EIO was judged longer than DIG. which was judged longer than $\mathrm{I}$.

These differences in the apparent duration of tachistoscopic flashes seem. then. to be indexing the operations of initial visual processing. The processing being indexed clearly apears to be prerecognition and appears to be heavily influenced by the familiarity of the presented stimulus forms: apparent contrast seems not to influence duration judgments unless its form is that of some minimally meaningful display such as a rectangular area or a verbally meaningful display. Differences in apparent durations of simple area differences are small. while initial processing of meaningful displays generates considerably larger differences in apparent duration.

\section{EXPERIMENT V}

The results of Experiment IV showed that the apparent duration differences for tachistoscopic presentations of I. DIG, and EIO were not due exclusively to apparent contrast and that the differences were not artifacts of initial experience in judging tachistoscopic flashes. However. these three stimulus displass were employed in both Experiments II and IV. and the possibility remains that the differences in apparent duration were less a function of stimulus familiarity than of the particular contours comprising the letters of these arrays. The fact that duration differences occurred with I and HIT in Experiment I does not recommend this possibility, but the hypothesis deserves a test. Experiment V provided one test of this possibility by preserving the contours of these displays. and the spatial relationships among these contours, while the familiarity of one of the displays was modified.

\section{Method}

Stimulus materials. Stimulus materials were the nine 1. DIG. and EIO slides emplosed in Experiments II and IV. presented for the same durations at the same luminance levels and by the same procedure. However. to leave the contours of the displays and the spatial relationships among contours unchanged (except for orientation), while familiarity of one stimulus display was modified. the DIG display was presented inverted (OIC). The following rationale dictated the operations of the experiment. If judged duration differences depended simply on particular contours and or their spatial relationships to each other, rather than the familiarity gained by experience in seeing a display presented in a particular orientation. then the relative judged durations of the three displays should replicate the findings of Experiments II and IV. On the other hand. if familiarity gained by past experience with a display in regular readable orientation were important in determining judged duration. then inverting DIG should eliminate the familiarity of the display. and its judged duration should not be less than that for EIO and clearly greater than that for $I$.

Design and Procedure. The design. procedure, and data anlusis duplicated those of the second task in Experiment IV.
Subjects. Ten volunteers from summer session psychology courses served as subjects and received course credit for participation. All had normal or corrected-to-normal vision.

\section{Results and Discussion}

Figure 4 presents mean judged duration for each stimulus form at each PCF level. Analysis of the $z^{\prime}$ scores revealed a highly significant main effect of stimulus form $[F(2.18)=44.87, p<.001]$ and of PCF $[F(2.18)=58.44 . p<.001]$ as well as a significant interaction between these variables $[\mathrm{F}(4.36)=5.24 . \mathrm{p}<.01]$. Applied to the interaction. Scheffé's test $(a=.05)$ confirmed what is evident in Figure 4. While there were no reliable differences between stimulus forms with $15 \%$ cells filled, the DId $=\mathrm{EIO}>\mathrm{I}$ differences were reliable at both $30 \%$ and $45 \%$ PCF levels.

While initial consideration of these results might suggest that the effect of inverting DIG was to prompt subjects to respond on the basis of a simple difference between the larger displays and the smaller displays, it should be noted that. in the first task of Experiment IV where subjects were responding to simple differences in display size, PCF did not differentially influence duration judgments. The interaction between stimulus form and PCF in Experiment $V$ strongly suggests that the effect of inverting DIG was to destroy subjects' familiarity with that form. In Experiments II and IV, the influence of PCF depended on differences in the familiarity of stimulus forms, with increases in PCF progressively increasing the differences in judged duration among

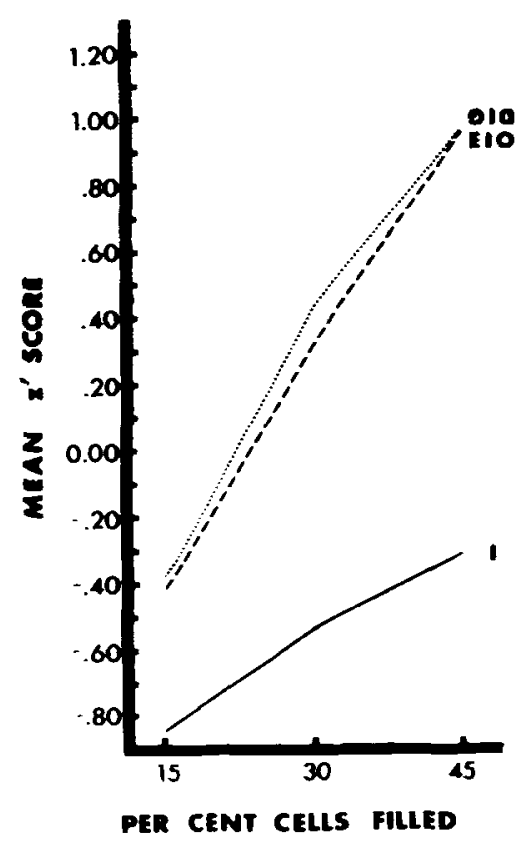

Figure 4. Mean $z$ score for I, DId, and EIO at each PCF kerd in Experiment V. 
stimulus forms. The observation that. in this experiment, increases in PCF progressively augmented the difference in judged duration for stimulus forms suggests that PCF was interacting with stimulus familiarity rather than stimulus display size.

\section{GENERAL DISCUSSION}

Collectively, the data of these experiments recommend the following conclusions: (1) judgments of the relative apparent duration of tachistoscopic presentations are systematically related to variations in the familiarity of the presented displays (Experiments I. II. IV. V). (2) the duration differences are unrelated to differences in apparent contrast except as contrast interacts with stimulus familiarity to augment effects of differences in familiarity on duration judgments (Experiment IV), and (3) duration judgments are more sensitivie to variations in stimulus familiarity under masking conditions which deter identification of the display elements than under no-mask conditions (Experiments II and III). The consistency of these findings recommends the relative apparent duration measure as a relatively sensitive index of the influence of such cognitive parameters as stimulus familiarity on early visual processing.

If the differences in relative apparent duration are indeed indexing the operations of early visual processing, they present important implications about the nature of that processing, at least when the presented displays are verbal and vary in familiarity. To briefly review the evidence that the indexed processing was prerecognition: Experiment II showed differences in the relative apparent duration to be clearer for the masked condition than for the no-mask condition and, under the masked condition, the apparent duration differences appeared to be more impressive to subjects; at least the lower response latency for the masked condition suggests that it was easier to make a decision between the apparent durations of paired masked flashes than to discriminate between unmasked flashes. These observations suggest that the differences in relative apparent duration are peculiar to the initial stage(s) of processing. and that, if presentation conditions permit full contact of the display elements with a central processor which prompts full recognition of the display elements, the initial duration differences are either bypassed or attenuated by the recognition response. An unpublished study by Cardillo (1973) buttresses this indication from the present data. Cardillo employed the pair comparisons procedure to test for differences in apparent durations between the smallest verbal unit (a single letter) and a nonverbal form. The letter $O$ with $25 \%, 50 \%$, and $75 \%$ of the matrix cells filled and a rectangular field of randomly dispersed dots were presented for relative duration judgments. Different groups of subjects judged paired

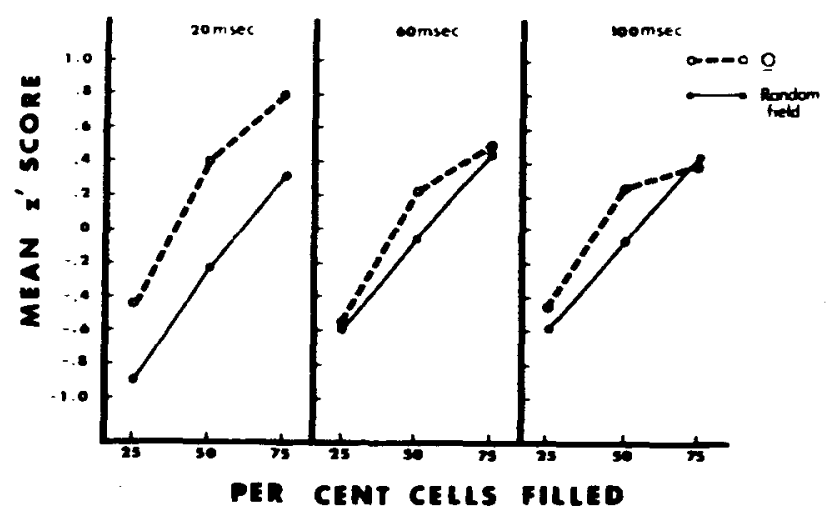

Figure 5. Mean z' score representing mean judged duration of the $O$ and the rectangular random field at each PCF level and each exposure duration (after Cardilio, 1973).

presentations of these displays when flash durations were 20,60 , and $100 \mathrm{msec}$. The important findings from Cardillo's study are presented in Figure 5.

These data make two salient points. First, the $O$ was judged to be of longer duration at all exposure durations. More importantly, the difference in apparent durations of the two forms was greatest for the 20-msec exposure, and the difference diminished as exposure duration was lengthened. Like the mask vs. no-mask comparisons of apparent durations and response latencies in Experiment II, Cardillo's data suggest the relative apparent duration differences to be peculiar to very early processing. When amount of processable information was limited to the briefest exposure (and presumably to initial processing stages), the difference in apparent duration between a meaningful verbal form and a simple rectangular area was impressive to subjects; when longer exposure durations permitted more extensive processing, the difference in apparent durations was considerably reduced.

An additional contribution of Cardillo's data to the present series of experiments should be noted. The reader will recall that, in the first task of Experiment IV, where the randomly dispersed dots presented only differences in the sizes of the rectangular stimulus area, duration judgments showed a weak. but statistically significant, difference in the apparent durations of the two display sizes. Cardillo's results extend that finding and show that when a meaningful verbal display $w$ as compared with a simple display form size, the more meaningful verbal display was judged to be of longer duration; apparent duration judgments appear to be more sensitive to cognitive differences among displays than to more primitive perceptual parameters such as display size.

Giver that these apparent duration differences reflect operations of initial visual processing, the consistent influence of stimulus familiarity contends that initial visual processing is, at least with verbal 
displays. an active process of beginning to establish contact between the input and the stored representation of some critical property of the display such as its degree of familiarity. While their interpretations of processing and coding differ in several regards. Posner (1969). Posner and Boies (19-1). Posner and Warren (1972). Shiffrin and Geisler (1973), and Wickens (1972) agree that at least some parameters of stimulus displays contact their memorial representations directly, automatically, and without the involvement of any attentional control mechanisms of a central processor. Posner and his colleagues (Cox. 1967; Eichelman, 1970) have emploved a same-different letter matching task and found the familiarity of English words to facilitate matching on the basis of physical identity of paired words without the necessity of the subject's making contact with the name codes for the displays. Similarly. Cox (1967) found simple visual pretraining with nonsense patterns to be as effective in facilitating same-different matching as was training involving verbal labels for the patterns. Wickens (1972) has employed an adaptation of the Brown-Peterson short-term memory paradigm with amount of release from proactive inhibition as the index of encoding and the automaticity of the contact between stimulus attributes and memory image. Some attributes or dimensions of words have been shown to be particularly effective in release from PI (semantic). while others have not (syntactic). Wickens suggests that increases in language experience may move an individual from an optional encoding situation to an automatic, and even obligatory, encoding situation with at least some attributes of verbal materials.

The effects of stimulus familiarity on relative apparent duration judgments are certainly consistent with the findings from Posner's and Wickens's studies. The particular merits of the relative judged duration measure that distinguish it from other measures of early processing are that it both demonstrates and provides a relatively sensitive index of familiarity effect on the ease of automatically initiating (but not necessarily completing) a contact between some dimensions of verbal materials and their memorial representations.

\section{REFERENCES}

BLFFARDI. L. Factors affecting the filled-duration illusion in the auditory. tactual. and visual modalities. Perception \& Psychophysics, 1971. 10, 292-294.

CARDILLO. L. J. The effects of processing varying amounts of information upon the perception of brief temporal intervals. Unpublished Master's thesis. Iowa State University, 1973.

$\operatorname{Cox} . N$. Effect of familiarization pretraining with random shapes on same-different judgment times. Unpublished master's thesss, Carleton University, 1967. Cited in Posner. M. I. Abstraction and the process of recognition. In G. H. Bower and J. T. Spence (Eds.), The psychology of learning and motivation (Vol.3). New York: Academic Press, 1969. Pp. 43-100.

EFron, R. An invariant characteristic of perceptual systems in the time domain. In S. Kornblum (Ed.). Attention and performance IV. New York: Academic Press, 1973. Pp. 713-736.

Eichelman, W. H. Familiarity effects in the simultaneous matching task. Journal of Experimental Psychology, 1970, 86, 275-282.

Frankenhaeuser, M. Estimation of time. Stockholm: Almquist Wicksells, 1959.

Ka RNEMAN. D. Method. findings and theory in studies of visual masking. Psychological Bulletin, 1968. 70, 404-425.

Lefton, L. A. Metacontrast: A review. Perception \& Psychophysics, 1973, 13, 161-171.

Liss. P. Does backward masking by visual noise stop stimulus processing? Perception \& Psychophysics, 1968, 4, 328-330.

Michon, J. A. Studies in subjective duration. II. Subjective time measurement during tasks with different information content. Acta Psychologica, 1965, 24, 205-219.

ORnsteIn, R. E. On the experience of time. Middlesex: Penguin. 1969.

Posner, M. I. Abstraction and the process of recognition. In G. H. Bower and J. T. Spence (Eds.), The psychology of learning and motivation (Vol. 3). New York: Academic Press, 1969. Pp. 43-100.

Posner, M. I., \& Bores, S. J. Components of attention. Psychological Review, 1971, 78, 391-408.

Posner, M. I., \& W WrRen, R. E. Traces, concepts, and conscious constructions. In A. W. Melton and E. Martin (Eds.), Coding processes in human memory. New York: Wiley, 1972. Pp. 25-43.

RAAB, D. H. Backward masking. Psychological Bulletin, 1963, 60, 118-129.

Reicher. G. M. Perceptual recognition as a function of meaningfulness of stimulus material. Journal of Experimental Psychologv. 1969. 81. 275.280.

Shiffrin, R. M., \& Geisler, W. S. Visual recognition in a theory of information processing. In R. L. Solso (Ed.), Contemporary issues in cognitive psychology: The Loyola symposium. New York: Wiley, 1973. Pp. 53-101.

SPERLiNG, G. Successive approximations to a model for short-term memory. Acta Psychologica, 1967, 27, 285-292.

STERnBerg, S. The discovery of processing stages: Extension of Donders' method. In W. G. Koster (Ed.), Attention and performance 1I. Amsterdam: North-Holland, 1969. Pp. 276-314.

TURVEY, M. T. On peripheral and central processes in vision: Inferences from an information-processing analysis of masking with patterned stimuli. Psychological Review', 1973, 80, 1-52.

Wheterer, D. D. Processes in word recognition. Cognitive Psychology, 1970, 1, 59-85.

Wickens, D. D. Characteristics of word encoding. In A. W. Melton and E. Martin (Eds.), Coding processes in human memory. New York: Wiley, 1972. Pp. 191-215.

WINER, B. I. Statistical principles in experimental design (2nd ed.). New York: McGraw-Hill, 1971.

Woodworth, R. S., \& ScBlosberg, H. Experimental psychology (Rev. ed.). New York: Holt, Rinehart, and Winston, 1954.

\section{NOTE}

1. Asant. L. L. Processing the $V$ in CVCs. In preparation.

(Received for publication August 2, 1974; revision received October $23,1974$. 